PROCEEDINGS OF THE

AMERICAN MATHEMATICAL SOCIETY

Volume 134, Number 8, Pages 2223-2228

S 0002-9939(06)07916-0

Article electronically published on March 14, 2006

\title{
SIMPLE REAL RANK ZERO ALGEBRAS WITH LOCALLY HAUSDORFF SPECTRUM
}

\author{
PING WONG NG
}

(Communicated by David R. Larson)

\begin{abstract}
Let $\mathcal{A}$ be a unital, simple, separable $C^{*}$-algebra with real rank zero, stable rank one, and weakly unperforated ordered $K_{0}$ group. Suppose, also, that $\mathcal{A}$ can be locally approximated by type I algebras with Hausdorff spectrum and bounded irreducible representations (the bound being dependent on the local approximating algebra). Then $\mathcal{A}$ is tracially approximately finite dimensional (i.e., $\mathcal{A}$ has tracial rank zero).

Hence, $\mathcal{A}$ is an $A H$-algebra with bounded dimension growth and is determined by $K$-theoretic invariants.
\end{abstract}

The above result also gives the first proof for the locally $A H$ case.

\section{INTRODUCTION}

In the $K$-theoretic classification program for simple unital separable stably finite nuclear $C^{*}$-algebras, a great deal of progress has been made for those algebras which have stable rank one, real rank zero, and weak unperforation in the ordered $K_{0^{-}}$ group (see, for example, 7, [13, 4], 1] and the last paragraph of [10]). One of the fundamental results in this direction is the work in 7, where Elliott and Gong classified (using $K$-theoretic invariants) all simple unital $A H$-algebras with bounded dimension growth and real rank zero.

We note that the class of algebras in [7] exhausts the current invariant for simple unital stably finite real rank zero nuclear $C^{*}$-algebras. Much work to date has been done to give classification results, for simple, nuclear, stably finite, real rank zero algebras, that do not assume that the $C^{*}$-algebras involved are $A H$-algebras (see, for example, [13] and the references therein).

Definition 1.1. Let $\mathcal{A}$ be a simple unital $C^{*}$-algebra. Then $\mathcal{A}$ is said to be tracially approximately finite dimensional (abbreviated by "TAF") if for every $\epsilon>0$, for every finite subset $\mathcal{F}$ of $\mathcal{A}$ and for every strictly positive element $a \in \mathcal{A}$, there is a projection $p$ which is Murray-von Neumann equivalent to a subprojection in the hereditary subalgebra generated by $a$ and there exists a finite dimensional $C^{*}$ subalgebra $\mathcal{B}$ of $\mathcal{A}$ such that: (a) $1_{\mathcal{A}}-p=1_{\mathcal{B}}$ where $1_{\mathcal{B}}$ is the unit of $\mathcal{B}$, (b) $\|x p-p x\|<\epsilon$ for every $x \in \mathcal{F}$, and $(\mathrm{c})\left(1_{\mathcal{A}}-p\right) x\left(1_{\mathcal{A}}-p\right)$ is within $\epsilon$ of an element of $\mathcal{B}$, for every $x \in \mathcal{F}$.

The term "tracial rank zero" is often used in place of "tracially approximately finite dimensional" (see, for instance, [12] and [13]). Hence, by definition, a simple,

Received by the editors November 21, 2003 and, in revised form, June 23, 2004.

2000 Mathematics Subject Classification. Primary 46L35.

(C)2006 American Mathematical Society Reverts to public domain 28 years from publication 2223 
unital $C^{*}$-algebra is $T A F$ if and only if it has tracial rank zero. (Indeed, Lin has a notion of tracial rank, which takes on values other than zero. For example, all nonreal rank zero, simple, unital $A H$-algebras, with bounded dimension growth, have tracial rank one; see [12].)

Lin has shown that the class of simple unital separable nuclear $T A F$ algebras which satisfy the universal coefficient theorem is exactly the class of [7] (see [13]).

Definition 1.2. Let $\mathcal{A}$ be a $C^{*}$-algebra. (a) Then $\mathcal{A}$ is said to be locally type I if for every $\epsilon>0$, for every finite subset $\mathcal{F}$ of $\mathcal{A}$, there is a separable type I $C^{*}$ subalgebra $\mathcal{B}$ of $\mathcal{A}$ such that every element of $\mathcal{F}$ is within $\epsilon$ of an element of $\mathcal{B}$. (b) If in (a), every (local approximating) type I $C^{*}$-algebra $\mathcal{B}$ has Hausdorff spectrum and there exists an integer $L$ (dependent on $\mathcal{B}$ ) such that every irreducible representation of $\mathcal{B}$ has dimension less than $L$, then $\mathcal{A}$ is said to have locally Hausdorff spectrum. (c) If in (a), every (locally approximating) type I $C^{*}$-algebra has the form $\bigoplus_{i=1}^{N} p_{i} \mathbb{M}_{n_{i}}\left(C\left(X_{i}\right)\right) p_{i}$, where each $X_{i}$ is a compact metric space and each $p_{i}$ is a projection in $\mathbb{M}_{n_{i}}\left(C\left(X_{i}\right)\right)$, then $\mathcal{A}$ is said to be locally $A H$.

We note that Dadarlat and Eilers have given an example of a (nonsimple) separable, unital, locally $A H C^{*}$-algebra which has real rank zero and stable rank one, but is not an $A H$-algebra (see [3]).

We also note that in [2], Dadarlat has shown that if $\mathcal{A}$ is a separable nuclear $C^{*}$-algebra which can be locally approximated by $C^{*}$-algebras which satisfy the universal coefficient theorem, then $\mathcal{A}$ also satisfies the universal coefficient theorem. Hence, every locally type I $C^{*}$-algebra satisfies the universal coefficient theorem.

In [15], Lin proved the following very interesting result (there are several proofs in the literature; other proofs can be found in [1, Corollary 7.11], [14] and [17, Theorem 5.16]):

Theorem 1.3. Let $\mathcal{A}$ be a unital separable simple locally type I $C^{*}$-algebra with real rank zero, stable rank one, weak unperforation in the $K_{0}$-group. Suppose also that the tracial simplex has countably many extreme points. Then $\mathcal{A}$ is TAF. By a theorem of Lin, this implies that $\mathcal{A}$ is an $A H$-algebra with bounded dimension growth and is determined by $K$-theoretic invariants.

We note that Lin's result requires a restriction on the tracial simplex of $\mathcal{A}$ (countably many extreme points). There have also been other interesting results in the literature which require this restriction on the tracial simplex (see, for example [1, the last paragraph of [10], 14, [15] and [17]).

In this paper, we remove the unique trace condition in Lin's result provided that the (local) type I algebras have Hausdorff spectrum and bounded irreducible representations.

Definition 1.4. $\mathcal{L C H}^{+}$is the class of simple unital separable $C^{*}$-algebras with real rank zero, stable rank one, weak unperforation in the ordered $K_{0}$-group, and having locally Hausdorff spectrum.

Theorem 1.5. Let $\mathcal{A}$ be a $C^{*}$-algebra in $\mathcal{L C H}^{+}$. Then $\mathcal{A}$ is TAF. Hence, by a theorem of Lin, $\mathcal{A}$ is an $A H$-algebra with bounded dimension growth and is determined by $K$-theoretic invariants.

Our result gives the first proof that a simple unital separable locally $A H C^{*}$ algebra with real rank zero, stable rank one, weak unperforation in the $K_{0}$ group is $T A F$, without any restriction on the tracial simplex. 
A modification of our argument gives a short alternative proof of the following result of Lin (which also follows from our result).

Theorem 1.6 (see [16]). Let $\mathcal{A}$ be a simple unital AH-algebra which has stable rank one, real rank zero and weakly unperforated $K_{0}$ group. Then $\mathcal{A}$ is $T A F$.

Note that in the hypothesis of the above result, it is not assumed that $\mathcal{A}$ has bounded dimension growth. Also, Lin's argument does not generalize to the locally $A H$ case.

In what follows, if $\mathcal{A}$ is a unital $C^{*}$-algebra, then $T(\mathcal{A})$ is the simplex of unital traces on $\mathcal{A}$.

\section{MAin RESUlt}

Proof of Theorem 1.5. Let $\left\{\mathcal{G}_{m}^{(1)}\right\}_{m=1}^{\infty}$ be an increasing sequence of finite subsets of $\mathcal{A}$ such that $\mathcal{A}=\overline{\bigcup_{m=1}^{\infty} \mathcal{G}_{m}^{(1)}}$. Let $f$ be the function on the unit interval [0,1] given by $f(t)=0$ for $t<1 / 2$ and $f(t)=1$ for $t \geq 1 / 2$. For each $m$, let $\mathcal{G}_{m}^{(2)}$ be the (finite) set of elements of $\mathcal{A}$ given by $\mathcal{G}_{m}^{(2)}{ }_{d f}\{f|a| /\|a\|): a \neq 0, a \in \mathcal{G}_{m}^{(1)}, f$ is continuous on the spectrum of $|a| /\|a\|\}$ (here, given $a \in \mathcal{A},|a|$ is the absolute value of $a$ and $\|a\|$ is the norm of $a$ ). Note that $\bigcup_{m=1}^{\infty} \mathcal{G}_{m}^{(2)}$ is dense in the set of projections of $\mathcal{A}$. Now for each $m$, let $\mathcal{G}_{m}={ }_{d f} \mathcal{G}_{m}^{(1)} \cup \mathcal{G}_{m}^{(2)}$. Since $\mathcal{A}$ is in $\mathcal{L C H}^{+}$, let $\left\{\mathcal{A}_{m}\right\}_{m=1}^{\infty}$ be a sequence of unital separable subalgebras of $\mathcal{A}$, with Hausdorff spectrum and bounded irreducible representations, such that for each $m, a$ is within a distance $1 / 2 m$ of an element, say $\phi_{m}(a)$, of $\mathcal{A}_{m}$ for every $a \in \mathcal{G}_{m}$. If $a$ is a projection in $\mathcal{G}_{m}^{(2)}$, we further require that $\phi_{m}(a)$ be a projection. Now, for each $m, \mathcal{A}_{m}$ need not be a continuous trace $C^{*}$-alegbra, but by [19, Theorem 4], $\mathcal{A}_{m}$ is "continuous trace" with respect to the normalized trace; that is, for each $a \in \mathcal{A}_{m}$, the map $\widehat{\mathcal{A}_{m}} \rightarrow \mathbb{R}$ given by $\pi \mapsto \operatorname{tr}(\pi(a))$ is continuous (where $t r$ is the unital, normalized trace on the image of $\pi$, and $\widehat{\mathcal{A}_{m}}$ is the spectrum space of irreducible representations of $\left.\mathcal{A}_{m}\right)$. But for each $m$, for each $p \in \mathcal{G}_{m}^{(2)}$, the map $\pi \mapsto \operatorname{tr}\left(\pi\left(\phi_{m}(p)\right)\right)$ can take on only finitely many (rational) values. Hence, $\widehat{\mathcal{A}_{m}}$ is the disjoint union of finitely many clopen sets such that for each $p \in \mathcal{G}_{m}^{(2)}$, the map $\pi \mapsto \operatorname{tr}\left(\pi\left(\phi_{m}(p)\right)\right)$ has constant value on each clopen set. Hence, for each $m, \mathcal{A}_{m}$ can be realized as a finite direct sum $\mathcal{A}_{m}=\bigoplus_{i=1}^{N_{m}} \mathcal{A}_{m, i}$ where each summand $\mathcal{A}_{m, i}$ has spectrum being one of the clopen sets. In particular, this means that for every $m$, for every projection $p \in \mathcal{G}_{m}^{(2)}$, for $1 \leq i \leq N_{m}$, the map $\pi \mapsto \operatorname{tr}\left(\pi\left(1_{\mathcal{A}_{m, i}} \phi_{m}(p) 1_{\mathcal{A}_{m, i}}\right)\right)$ is constant on the spectrum $\widehat{\mathcal{A}_{m, i}}$ (where "tr", as always, denotes the unital normalized trace on the image of $\pi$ ). We may assume that $1_{\mathcal{A}_{m}}=1_{\mathcal{A}}$ for every $m$. Let $\mathcal{B}={ }_{d f} \sum_{m=1}^{\oplus} \sum_{i=1}^{\oplus N_{m, i}} \mathcal{A}_{m,} \sum_{l=1}^{\oplus} \mathcal{B}_{l}$. Then the multiplier algebra of $\mathcal{B}$ is $\mathcal{M}(\mathcal{B})=\prod_{l=1}^{\infty} \mathcal{B}_{l}$ such that each $\mathcal{B}_{l}$ is one of the $\mathcal{A}_{m, i} s$.

For $a \in \bigcup_{m=1}^{\infty} \mathcal{G}_{m}$ and for each strictly positive integer $l$, let $(a, l)$ be an element $b \in \mathcal{B}_{l}$ defined in the following manner: Suppose that $\mathcal{B}_{l}$ is the summand $\mathcal{A}_{m, i}$ of $\mathcal{M}(\mathcal{B})$. If $a$ is in $\mathcal{G}_{m}$, then let $(a, l)$ be $1_{\mathcal{B}_{l}} \phi_{m}(a) 1_{\mathcal{B}_{l}}$. Otherwise, let $(a, l)$ be zero.

We may assume that for every integer $l,\left(1_{\mathcal{A}}, l\right)=1_{\mathcal{B}_{l}}$. We have an $*$-homomorphism $\Gamma: \mathcal{A} \rightarrow \mathcal{M}(\mathcal{B}) / \mathcal{B}$ which is defined as follows: suppose that $a \in \mathcal{A}$. Let $\left\{a_{n}\right\}_{n=1}^{\infty}$ be a sequence in $\bigcup_{m=1}^{\infty} \mathcal{G}_{m}$ which converges to $a$. Then we let $\Gamma(a)=_{d f}$ $\lim _{n \rightarrow \infty}\left(a_{n}, l\right) / \mathcal{B}$. One can check that $\Gamma$ is indeed a well-defined $*$-homomorphism. 
Now since $\mathcal{A}$ is simple, $\Gamma$ is either injective or the zero map. Since $\left(1_{\mathcal{A}}, l\right)=1_{\mathcal{B}_{l}}$ for every $l, \Gamma$ is unital and hence must be injective.

For each $l$, let $\tau_{l}$ be a unital trace on $\mathcal{B}_{l}$ obtained by a point evaluation on $\hat{\mathcal{B}}_{l}$, the spectrum of $\mathcal{B}_{l}$ (that is, $\tau_{l}$ is obtained by composing an irreducible representation of $\mathcal{B}_{l}$ with the usual unital trace on matrices).

Let $\epsilon>0$ and a finite subset $\mathcal{F}$ of $\mathcal{A}$ be given. To show that $\mathcal{A}$ is $T A F$, we need to prove that there is a projection $p \in \mathcal{A}$ and there is a finite dimensional $C^{*}$-subalgebra $\mathcal{C}$ of $\mathcal{A}$, with $1_{\mathcal{C}}=1-p$, such that:

(1) $\sup _{r \in T(\mathcal{A})} \tau(p)<\epsilon$,

(2) $\|p f-f p\|<\epsilon$ for every $f \in \mathcal{F}$, and

(3) $(1-p) f(1-p)$ is within $\epsilon$ of an element of $\mathcal{C}$ for every $f \in \mathcal{F}$.

So, let $\epsilon$ and $\mathcal{F}$ be given as above. To simplify notation, we may assume that each element of $\mathcal{F}$ has norm less than or equal to one (adjust $\epsilon$ if necessary).

Claim. There is a strictly positive integer $L$ such that for each $l \geq L$, there is a projection $p_{l}$ in $\mathcal{B}_{l}$ and a finite dimensional $C^{*}$-subalgebra $\mathcal{C}_{l}$ of $\mathcal{B}_{l}$ with $1_{\mathcal{C}_{l}}=1_{\mathcal{B}_{l}}-p_{l}$ such that:

(1) if $\mathcal{B}_{l}$ is the summand $\mathcal{A}_{m, i}$, then $p_{l}$ has the form $1_{\mathcal{B}_{l}} \phi_{m}(p) 1_{\mathcal{B}_{l}}$ for some projection $p \in \mathcal{G}_{m}^{(2)}$,

(2) $\tau_{l}\left(p_{l}\right)<\epsilon / 100$

(3) $\left\|p_{l} f-f_{p_{l}}\right\|<\epsilon / 100$ for every $f \in \mathcal{F}$, and

(4) $1_{\mathcal{C}_{l}} f 1_{\mathcal{C}_{l}}$ is within $\epsilon / 100$ of an element of $\mathcal{C}_{l}$ for every $f \in \mathcal{F}$.

Now suppose, to the contrary, that the claim is not true. Let $\left\{l_{\alpha}\right\}_{\alpha \in I}$ be a subnet of the sequence of positive integers such that for each $\alpha \in I$, the statement of the claim does not hold for $l=l_{\alpha}$. Now for each integer $k$, let $\tilde{\tau}_{k}$ be the trace on $\mathcal{M}(\mathcal{B})=\prod_{l=1}^{\infty} \mathcal{B}_{l}$ given by $\tilde{\tau}_{k}\left(\left(a_{l}\right)_{l=1}^{\infty}\right)=\tau_{k}\left(a_{k}\right)\left(\tau_{k}\right.$ is defined two paragraphs before the claim, and $a_{l} \in \mathcal{B}_{l}$ for every $\left.l\right)$. Now since $T(\mathcal{M}(\mathcal{B}))$ is $w^{*}$-compact, the net $\left\{\tilde{\tau}_{l_{\alpha}}\right\}_{\alpha \in I}$ has a converging subnet. For simplicity, let us assume that $\left\{\tilde{\tau}_{l_{\alpha}}\right\}_{\alpha \in I}$ converges to, say $\tilde{\tau}$. Note that $\tilde{\tau}$ induces a trace on $\mathcal{M}(\mathcal{B}) / \mathcal{B}$, which we also denote by " $\tilde{\tau}$ ". Since $\Gamma: \mathcal{A} \rightarrow \mathcal{M}(\mathcal{B}) / \mathcal{B}$ is a unital $*$-embedding, $\tilde{\tau} \circ \Gamma$ is a tracial state on $\mathcal{A}$. For simplicity, we will also denote $\tilde{\tau} \circ \Gamma$ by " $\tilde{\tau} "$.

Note that the argument of Theorem 1.3 actually works for any (arbitrary) single trace (see either [1, Corollary 7.11], [15] or [17, Theorem 5.16], and in the locally $A H$ case, an elementary proof can be obtained using the argument in the last section of 11). Hence, we have that there exists a projection $q \in \mathcal{A}$ and a finite dimensional $C^{*}$-subalgebra $\mathcal{D}$ of $\mathcal{A}$ with $1_{\mathcal{D}}=1-q$ such that:

(1) $\tilde{\tau}(q)<\epsilon / 1000$,

(2) $\|q f-f q\|<\epsilon / 1000$ for every $f \in \mathcal{F}$, and

(3) $(1-q) f(1-q)$ is within $\epsilon / 1000$ of an element of $\mathcal{D}$ for every $f \in \mathcal{F}$.

Now by our choices of the $\mathcal{A}_{m} \mathrm{~s}$ and $\mathcal{G}_{m}^{(2)} \mathrm{s}$, there is a positive integer $M>0$, and there is a sequence $\left\{\epsilon_{m}\right\}_{m=1}^{\infty}$ of positive real numbers converging to zero, such that for each $m \geq M$, we have the following:

(a) There is a matrix algebra, say $\mathcal{D}_{m}$, which is a subalgebra of $\mathcal{A}_{m}$, and there is a unitary element $U$ of $\mathcal{A}$ such that (i) $\mathcal{D}_{m}=U \mathcal{D} U^{*}$, and (ii) $U$ is within $\epsilon_{m}$ of $1_{\mathcal{A}}$.

(b) $U q U^{*}=1_{\mathcal{A}}-1_{\mathcal{D}_{m}}$, and $1_{\mathcal{A}}-1_{\mathcal{D}_{m}}$ is an element of $\mathcal{G}_{m}^{(2)}$. (Recall that $1_{\mathcal{A}}=1_{\mathcal{A}_{m}}$.)

(c) $\left(1_{\mathcal{A}}-1_{\mathcal{D}_{m}}\right) a$ is within $\epsilon / 500$ of $a\left(1_{\mathcal{A}}-1_{\mathcal{D}_{m}}\right)$, for every $a \in \mathcal{F}$. 
Now suppose that for each $m, \mathcal{A}_{m}=\bigoplus_{l=L_{m}}^{L_{m+1}-1} \mathcal{B}_{l}$ (so $\mathcal{B}_{L_{m}+k}=\mathcal{A}_{m, k+1}$ and $\left.L_{m+1}-L_{m}=N_{m}\right)$. And suppose that for $m \geq M, 1_{\mathcal{A}}-1_{\mathcal{D}_{m}}=q_{L_{m}} \oplus q_{L_{m}+1} \oplus$ $q_{L_{m}+2} \oplus \cdots \oplus q_{L_{m+1}-1}$, where $q_{L_{m}+k}$ is a projection in $\mathcal{B}_{L_{m}+k}$, for each $k$. Then $\Gamma(q)=\left(0,0, \ldots, 0, q_{L_{M}}, q_{L_{M}+1}, q_{L_{M}+2}, \ldots\right) / \mathcal{B}$, where $q_{L_{M}}$ is in the $L_{M}$ th position, and where we view $\mathcal{B}$ as $\mathcal{B}=\sum_{l=1}^{\oplus} \mathcal{B}_{l}$.

By the definition of $\tilde{\tau}$, and since $\tilde{\tau}(q)<\epsilon / 1000$, we must have that $\lim _{\alpha} \tau_{l_{\alpha}}\left(q l_{\alpha}\right)<$ $\epsilon / 1000$. Choose $\alpha_{0}$ such that for $\alpha \geq \alpha_{0}, \tau_{l_{\alpha}}\left(q_{l_{\alpha}}\right)<\epsilon / 1000$. Let $m_{0}$ be the integer such that $\mathcal{B}_{l_{\alpha_{0}}}$ comes from $\mathcal{A}_{m_{0}}$. Choosing $\alpha_{0}$ "large" enough if necessary, we may assume that $m_{0} \geq M$ and $\epsilon_{m_{0}}<\epsilon / 1000$. Hence, taking $l=l_{\alpha_{0}}, m=m_{0}$, $\mathcal{C}_{l}=1_{\mathcal{B}_{l_{\alpha_{0}}}} \mathcal{D}_{m_{0}} 1_{\mathcal{B}_{l_{\alpha_{0}}}}$, and $p_{l}=q_{l_{\alpha_{0}}}$, we have that clauses (1)-(4) of the Claim are satisfied for $l=l_{\alpha_{0}}$. This is a contradiction. Hence, the Claim must be true.

So let $L$ and $\mathcal{C}_{l}$ and $p_{l} \forall l \geq L$ be as in the Claim. Recalling our definition of $\mathcal{B}$ and the $\mathcal{B}_{l} \mathrm{~s}$, suppose that $m, N_{m}$ are integers such that $\mathcal{B}_{L+j}=\mathcal{A}_{m, j}$ for $1 \leq j \leq N_{m}$ and $\mathcal{A}_{m}=\bigoplus_{j=1}^{N_{m}} \mathcal{A}_{m, j}=\bigoplus_{j=1}^{N_{m}} \mathcal{B}_{L+j}$. Let $r={ }_{d f} \bigoplus_{j=1}^{N_{m}} p_{L+j}$ and $\mathcal{E}={ }_{d f} \bigoplus_{j=1}^{N_{m}} \mathcal{C}_{L+j}$. Then (a) $1_{\mathcal{E}}=1-r$, (b) $\|r f-f r\|<\epsilon$ for every $f \in \mathcal{F}$, and (c) $(1-r) f(1-r)$ is within $\epsilon$ of an element of (the finite-dimensional $C^{*}$-algebra) $\mathcal{E}$ for every $f \in \mathcal{F}$.

Also, by clause (1) of the Claim, it follows that for $1 \leq j \leq N_{m}$, the map on $\widehat{\mathcal{B}_{L+j}}$ (the spectrum of $\left.\mathcal{B}_{L+j}\right)$, given by $\pi \mapsto \operatorname{tr}\left(\pi\left(p_{L+j}\right)\right.$ ), is a constant rational-valued function (here, $t r$ is the unital trace on matrices). Hence, since $\tau_{L+j}\left(p_{L+j}\right)<\epsilon / 100$ for $1 \leq j \leq N_{m}$, it follows that $\tau(r)<\epsilon$ for every $\tau \in T(\mathcal{A})$.

So since $\epsilon$ and $\mathcal{F}$ are arbitrary, $\mathcal{A}$ is $T A F$.

\section{REFERENCES}

[1] N. Brown, Invariant means and finite representation theory of $C^{*}$-algebras, preprint. Available online at http://arxiv.org/abs/math.OA/0304009.

[2] M. Dadarlat, Some remarks on the universal coefficient theorem in KK-theory, Constanţa, (2003), 65-74. MR2018224(2004j:19005)

[3] M. Dadarlat and S. Eilers, Approximate homogeneity is not a local property, J. Reine Angew. Math., 507, (1999), 1-13. MR1670254 (2000b:46094)

[4] M. Dadarlat and S. Eilers, On the classification of nuclear $C^{*}$-algebras, Proc. London Math. Soc. (3), 85, (2002), no. 1, 168-210. MR1901373 (2003d:19006)

[5] M. Dadarlat and G. Gong, A classification result for approximately homogeneous $C^{*}$-algebras of real rank zero, Geom. Funct. Anal., 7, (1997), no. 4, 646-711. MR1465599 (98j:46062)

[6] J. Dixmier, $C^{*}$-algebras, Paris, 1964; North Holland, Amsterdam/New York/Oxford.

[7] G. Elliott and G. Gong, On the classification of $C^{*}$-algebras of real rank zero II, Ann. of Math. (2), 144, (1996), no. 3, 497-610. MR1426886 (98j:46055)

[8] G. Elliott, G. Gong, L. Li, On the classification of simple inductive limit $C^{*}$-algebras, II: the isomorphism theorem, preprint.

[9] J. M. G. Fell, The structure of algebras of operator fields, Acta Math., 106, (1961), 233-280. MR0164248 (29:1547)

[10] E. Kirchberg and W. Winter, Covering dimension and quasidiagonality, Internat. J. Math., 15, (2004), 63-85. MR2039212 (2005a:46148)

[11] H. Lin, Homomorphisms from $C^{*}$-algebras of continuous trace, Math. Scand., 86, (2000), no. 2, 249-272. MR:1754997(2001b:46088)

[12] H. Lin, The tracial topological rank of $C^{*}$-algebras, Proc. London Math. Soc. (3), 83, (2001), no. 1, 199-234. MR.1829565 (2002e:46063)

[13] H. Lin, Classification of simple $C^{*}$-algebras of tracial topological rank zero, Duke Math. J., 125, (2004), 91-119. MR2097358

[14] H. Lin, Classification of simple $C^{*}$-algebras with unique traces, Amer. J. Math., 120, (1998), no. 6, 1289-1315. MR1657178(2000c:46106)

[15] H. Lin, Locally type I simple TAF $C^{*}$-algebras, preprint.

[16] H. Lin, Simple AH-algebras of real rank zero, preprint. To appear in the Proceedings of the AMS. 
[17] H. Lin, Traces and simple $C^{*}$-algebras with tracial topological rank zero. J. Reine Angew. Math., 568, (2004), 99-137. MR2034925 (2005b:46122)

[18] I. Raeburn and D. Williams, Morita equivalence and continuous trace $C^{*}$-algebras, Mathematical Surveys and Monographs, 60, AMS, Providence, RI, 1998. MR.1634408 (2000c:46108)

[19] N. B. Vasil'ev, $C^{*}$-algebras with finite-dimensional irreducible representations, Uspehi Mat. Nauk, 21, (1966), no. 1, (127), 135-154. MR0201994 (34:1871)

Department of Mathematics and Statistics, University of New Brunswick, Fredericton, New Brunswick, Canada E3B 5A3

E-mail address: pwn@erdos.math.unb.ca

Current address: The Fields Institute for Research in Mathematical Sciences, 222 College Street, Toronto, Ontario, Canada M5T 3J1 